\title{
A study of the composition and energy spectra of anomalous cosmic rays using the geomagnetic field
}

\author{
R. A. Mewaldt, J. R. Cummings, R. A. Leske, R. S. Selesnick, and E. C. Stone \\ California Institute of Technology, Pasadena
}

T. T. von Rosenvinge

Goddard Space Flight Center Code 661, Greenbelt, Maryland

\begin{abstract}
We use instrumentation on SAMPEX and the Earth's field as a magnetic rigidity filter in a "double spectrometer" approach to measure the composition and energy spectra of anomalous cosmic rays (ACRs) with $\mathrm{Z} \geq 6$. A "pure" sample of anomalous cosmic ray $\mathrm{C}, \mathrm{N}, \mathrm{O}$, and $\mathrm{Ne}$ is obtained, with no significant evidence for other species. The bulk of ACRs are now known to be singly-charged, and the geomagnetic filter allows their energy spectra to be measured to higher energies than before. The anomalous oxyg'n spectrum is found to extend to at least $\sim 100 \mathrm{MeV} /$ nuc, which has implications for models of the acceleration of these ions.
\end{abstract}

\section{Introduction}

Anomalous cosmic rays (ACRs) are a low-energy component of interplanetary particles that include the elements $\mathrm{H}, \mathrm{He}, \mathrm{C}, \mathrm{N}, \mathrm{O}, \mathrm{Ne}$, and $\mathrm{Ar}$ (see, e.g., reviews by Klecker 1995, and Simpson 1995). They are now known to originate from interstellar neutral particles that have been swept into the heliosphere, ionized by solar UV or charge exchange with the solar wind, convected into the outer heliosphere, and then accelerated to energies of $\sim 10 \mathrm{MeV} /$ nuc and more (Fisk, Ramaty, and Koslovsky 1974). It is commonly assumed that the bulk of ACR acceleration takes place at the solar wind termination shock (Pesses, Jokipii, an.d Eichler 1981). A key prediction of this model, now established experimentally, is that ACRs should be singlyionized. In particular, Klecker et al. (1995) find that $<10 \%$ of $\sim 10 \mathrm{MeV} /$ nuc $\mathrm{ACR}$ oxygen has an ionic charge $\mathrm{Q} \geq 2$.

Being singly-charged, ACRs have a much greater magnetic rigidity (at a given energy/nuc) than either galactic cosmic rays (GCRs), which are essentially fully stripped, or solar energetic particles (SEPs), which have charge states characteristic of coronal temperatures of $2 \times 10^{6}{ }^{\circ} \mathrm{K}$ (Luhn et al. 1984; Leske et al. 1995a). As a result, ACRs can be observed to much lower invariant latitude with a polar orbiting spacecraft (Adams et al. 1991, Cummings et al. 1993, Klecker et al. 1995). The invariant latitude is $\Lambda=\cos ^{-1}\left[(1 / \mathrm{L})^{1 / 2}\right]$, where $L$ is the McIlwain $L$ parameter. In this paper we use the geomagnetic field as a magnetic rigidity filter to obtain a "pure" sample of ACRs, and then measure their compositic., and energy spectra over a broad energy range.

Copyright 1996 by the American Geophysical Union.

Paper number 96GL00423

0094-8534/96/96GL-00423\$03.00
Previously, Adams et al. (1991) used a similar approach to obtain orbit-averaged spectra of ACR oxygen using passive detectors flown on a series of Cosmos satellites. Our study differs from that of Adams et al. because the sub-second time resolution provided by SAMPEX allows the selection of data from specific latitude intervals. A preliminary version of this work appears in Mewaldt et al. (1995).

\section{Observations}

The observations were made with the Mass Spectrometer Telescope (MAST; see Cook et al. 1993) on the polar-orbiting (82 ${ }^{\circ}$ inclination) SAMPEX satellite (Baker et al. 1993) from 6 July 1993 to 7 January 1995. The measured kinetic energy vs. invariant latitude for quiet-time oxygen events is shown in Figure 1, limited to events observed during those periods when the daily-average flux of 8 to $15 \mathrm{MeV} / \mathrm{nuc} \mathrm{He}$ was $<4 \times 10^{-4}$ per $\mathrm{cm}^{2} \mathrm{sec} \cdot \mathrm{sr}$. This criterion eliminated $\sim 10 \%$ of the available days.

Three distinct particle populations are evident in Figure 1. At high latitudes $\left(\Lambda>65^{\circ}\right)$, there is a mixture of GCR and ACR oxygen with the ACRs more abundant at the lowest energies ( $<30 \mathrm{MeV} / \mathrm{nuc}$ ), and GCRs more abundant at higher energies. At mid-latitudes $\left(\Lambda \approx 50^{\circ}\right.$ to $60^{\circ}$ ), fully-stripped GCRs are not allowed but singly-charged ACR oxygen nuclei have access because of their greater magnetic rigidity. Finally, at luw latitudes $\left(\Lambda \leq 50^{\circ}\right)$ there is a radiation belt composed of ions that originate from ACRs that have lost their electrons in the upper atmosphere and then been trapped in the geomagnetic field because of their resulting lower rigidity (Blake and Friesen 1977; Grigorov et al. 1991; Cummings et al. 1993).

Also shown in Figure 1 are the boundaries used to isolate a pure sample of ACRs. The upper boundary at $\Lambda \approx 52^{\circ}$ to $65^{\circ}$ is designed to filter out GCRs and SEPs. It is based on the empirical geomagnetic cutoff vs. latitude relation determined by Leske et al. (1995a) from observations of solar energetic particle $\mathrm{He}$ and $\mathrm{C}$, lowered by an additional $20 \%$ to guard against contamination by fully-stripped nuclei during geomagnetic storms that might temporarily lower the cutoff. This is equivalent to a latitude restriction $\sim 4^{\circ}$ below the vertical geomagnetic cutoff. The boundary location is calculated for the measured nuclear charge, mass, and kinetic energy of each particle, and only particles observed below this boundary are accepted.

To filter out trapped anomalous cosmic rays we use the observation that they are characterized by an "adiabaticity" parameter $(\varepsilon)$, such that $\varepsilon Q_{s}<0.8$ (Selesnick et al. 1995). Here $\varepsilon=0.000052 \cos ^{-4}(\Lambda)\left(A / Q_{s}\right)\left[E^{2}+2 M_{p} E\right]^{1 / 2}$, where $A$ is the number of nucleons, $Q_{s}$ is the ionic charge state just after stripping in the initial atmospheric encounter, $\mathrm{E}$ is kinetic 


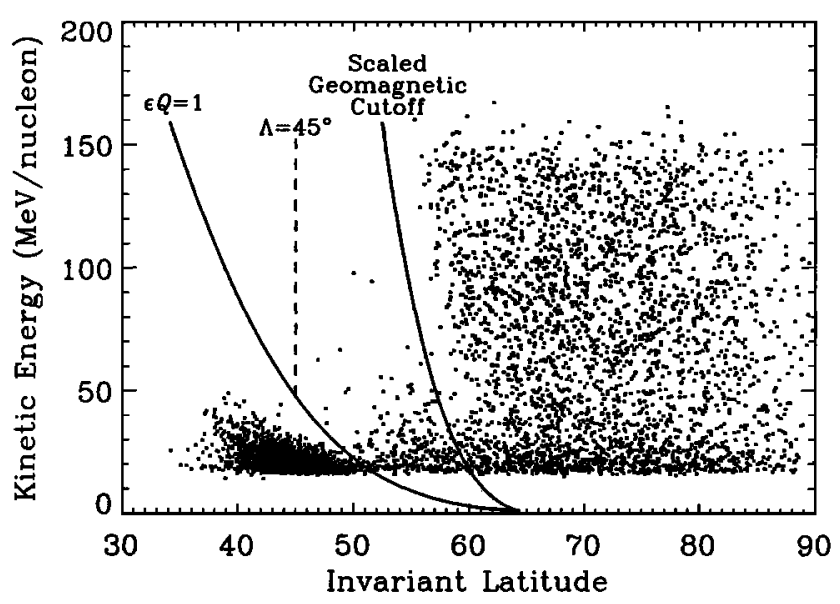

Figure 1. Measured energy/nucleon vs. invariant latitude for quiet-time oxygen events observed by the MAST instrument on SAMPEX from 6 July 1992 to 7 January 1995. The study includes events between the solid lines, with the added requirement that $\Lambda>45^{\circ}$ (dashed line). Trapped ACRs are seen below $50^{\circ}$.

energy in $\mathrm{MeV} / \mathrm{nuc}$, and $\mathrm{M}_{\mathrm{p}}$ is the proton rest mass in $\mathrm{MeV}$. Note that $\varepsilon Q_{s}$ is a constant of motion as long as $E$ and $L$ [defined as $1 / \cos ^{2}(\Lambda)$ ] remain constant. Following Selesnick et al. (1995) we require $\varepsilon Q_{s}>1$. In addition, we require $\Lambda>45^{\circ}$ to ensure that all ACRs with $>15 \mathrm{MeV} /$ nuc have good access to SAMPEX, which is generally zenith oriented for $\Lambda>45^{\circ}$, but has a more varied orientation at lower latitudes. Using a Monte-Carlo approach, we have traced a large number of particle trajectories in the IGRF 1992 magnetic ficld and verified that the actual SAMPEX pointing directions for $\Lambda>45^{\circ}$ result in an acceptance of at least $90 \%$ for all singly-charged ACRs with $\mathrm{Z} \geq 6$ and $\mathrm{E} \geq 15 \mathrm{MeV} /$ nuc.

The quiet-time composition of $\mathrm{Z} \geq 6$ ions measured at midlatitude, shown in Figure 2, shows evidence for ACR C, N, O, and $\mathrm{Ne}$, but no significant evidence for other species. The resulting composition, including corrections for the differing energy interval covered by each species, is shown in Table 1. Note that there is good consistency with the ACR composition observed by Voyager in 1987 at 23 AU. Voyager also observes ACR Ar at low energies (Cummings and Stone 1988), but the number of ACR Ar events that would be expected to be observed by MAST during this time interval is less than one.

ACR energy spectra from the mid-latitude region are compared with interplanetary spectra obtained at $\Lambda>65^{\circ}$ in Figure 3. It is interesting that the mid-latitude spectra appear to be consistent with a power law shape, with a spectral index of approximately -3.5 to -4 . While it is possible that interplanetary transport to $1 \mathrm{AU}$ may have modified the slope of the accelerated spectra, one would not expect this to be a significant effect because of the relatively large rificitities of these ions ( $\sim 3$ to $7 \mathrm{GV}$ for 16 to $100 \mathrm{MeV} /$ nuc singly charged oxygen).

The data in Figure 3 show that the energy spectra of ACR N and $\mathrm{Ne}$ extend to $>50 \mathrm{MeV} /$ nuc (the highest energy $\mathrm{N}$ and $\mathrm{Ne}$ had 57 and $68 \mathrm{MeV} / \mathrm{nuc}$, respectively), while that of ACR oxygen extends to at least $100 \mathrm{MeV} / \mathrm{nuc}$ (where two $\mathrm{O}$ iolss were observed, well below cutoff, on geomagnetically quiet days). This means that the ACR accelerator (presumably the termination shock) must be capable of accelerating particles

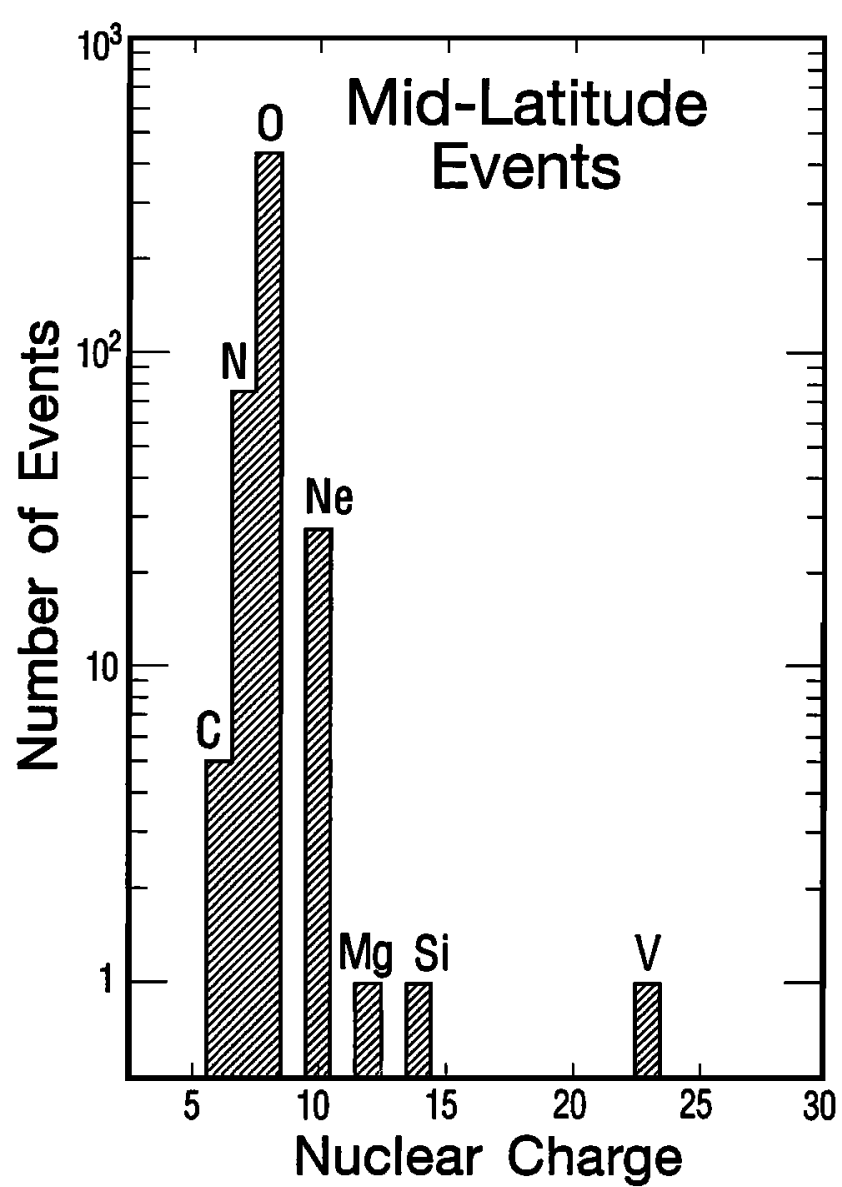

Figure 2. Composition of events with energies greater than $\sim 15 \mathrm{MeV} /$ nuc observed at mid-latitudes. No corrections have been applied for energy or latitude interval differences. The $\mathrm{Mg}$ and $\mathrm{Si}$ events both had an observed energy of $\sim 30$ $\mathrm{MeV} /$ nuc, while the single $\mathrm{V}$ event had $\sim 200 \mathrm{MeV} /$ nuc.

to at least $1.6 \mathrm{GeV}$, corresponding in the model of Jokipii (1990) to $400 \mathrm{MeV} / \mathrm{nuc}$ for ACR He and $1.6 \mathrm{GeV}$ for ACR hydrogen.

\section{Discussion}

The acceleration of singly-charged ACRs to energies $>1$ $\mathrm{GeV}$ must occur in a time less than the lifetime against electron stripping if ACRs are mainly singly ionized. Based on arguments in Adams and Leising (1991), Jokipii (1992) obtained a lifetime against stripping of $\sim 5$ years at $\sim 100 \mathrm{AU}$ for $10 \mathrm{MeV} /$ nuc ACRs. He further estimated that diffusive

Table 1. Anomalous Cosmic Ray Abundances

\begin{tabular}{|c|c|c|}
\hline Element & $\begin{array}{c}\text { Mid-Latitude } \\
\text { ACRs } \\
\text { >17 MeV/nuc }\end{array}$ & $\begin{array}{c}\text { Voyager-2 } \\
\text { at } 23 \mathrm{AU} * \\
16-30 \mathrm{MeV} / \mathrm{nuc}\end{array}$ \\
\hline $\begin{array}{c}\mathbf{C} \\
\mathbf{N} \\
\mathbf{O} \\
\text { Ne } \\
\text { All Others }\end{array}$ & $\begin{array}{c}0.014 \pm .009 \\
0.19 \pm .03 \\
1 \\
0.06 \pm .02 \\
<0.01\end{array}$ & $\begin{array}{c}0.020 \pm .004 \\
0.194 \pm .013 \\
1 \\
0.048 \pm .006\end{array}$ \\
\hline
\end{tabular}

*Selesnick et al. (1995) 


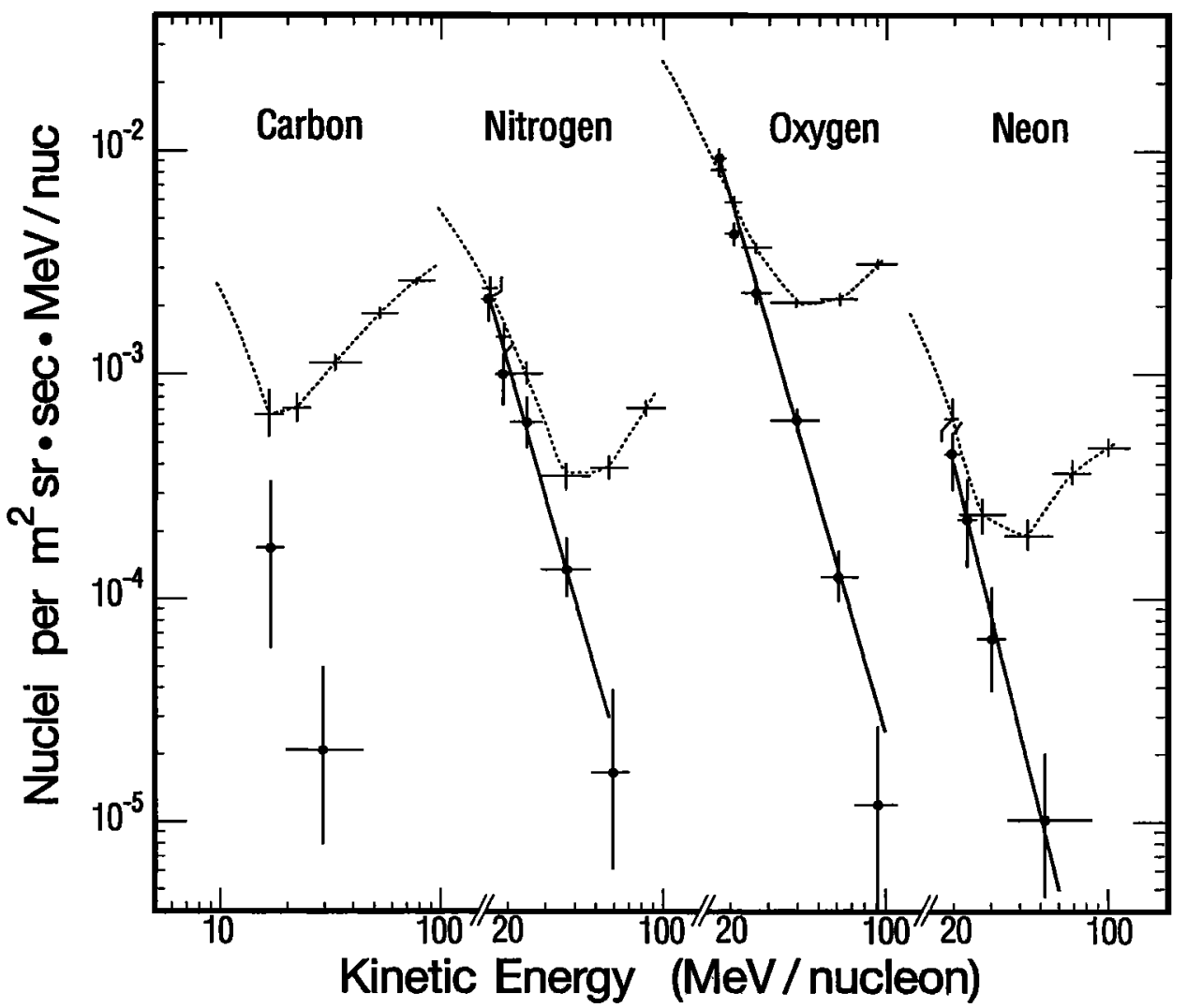

Figure 3. Energy spectra of ACRs measured at mid-latitudes, compared to interplanetary spectra obtained at $\Lambda>65^{\circ}$ (dashed lines). Below $\sim 15 \mathrm{MeV} /$ nuc the dashed lines have been extended to illustrate the shape of the 1992-1993 spectra observed by Mewaldt et al. (1993). The solid lines are power law fits to the mid-latilude spectra, with spectral indices for $\mathrm{N}, \mathrm{O}$, and $\mathrm{Ne}$ of -3.56 $\pm 0.43,-3.45 \pm 0.15$, and $-4.17 \pm 0.85$, respectively.

shock acceleration to $10 \mathrm{MeV} /$ nuc at $\sim 100 \mathrm{AU}$ would require a minimum of $\sim 0.5$ years for a perpendicular shock (the expecicú geometry of the termination shock), and at least four years for a quasi-parallel shock (see also Klecker 1995). Corresponding acceleration times for $100 \mathrm{MeV} /$ nuc ACRs would be a factor of 10 longer, in which case the integrated probability of stripping one or more electrons would be considerably greater than at lower energies because of the much greater pathlength traversed. Thus, the acceleration and stripping times appear to be comparable, suggesting that higher energy ACRs may not all be singly-charged. If this is the case, measurements of the charge states of ACRs with higher energy than the $\sim 10$ $\mathrm{MeV} /$ nuc particles studied by Klecker et al. (1995) and Adams et al. (1991) may provide insight into the acceleration times of ACRs in the heliosphere.

The possibility that a significant fraction of higher energy ACRs may have a charge $Q>1$ may also provide a partial explanation of how ACRs are accelerated to energies $>1 \mathrm{GeV}$. According to Jokipii (1990), the energy gain $\Delta \mathrm{E}$ by an ion with an electrical charge Qe at a quasi-perpendicular shock is approximately the electrostatic potential $\Delta \phi$ gained in drifting along the shock face: $\Delta \mathrm{E} \approx \mathrm{Qe} \Delta \phi$. He finds $\Delta \mathrm{E} \approx 240 \mathrm{MeV}$ for the termination shock, assuming $Q=1$, and predicts that there should be a steepening of the accelerated ACR spectra at energies $>240 \mathrm{MeV}$ (corresponding to $\sim 15 \mathrm{MeV} /$ nuc for ACR oxygen). While the expected location of this break is at the lower edge of the MAST energy interval, data from the HILT sensor on SAMPEX do show a flattening of the spectrum below $15 \mathrm{MeV} / \mathrm{nuc}$ (see Mewaldt et al. 1993).
According to the above relation for $\Delta \mathrm{E}$, singly-charged ACRs that happen to be stripped of additional electrons in the course of their acceleration will gain more energy than those that remain singly-charged. Thus the likely existence of ACRs with $Q>1$ may help explain the relatively large flux of ACRs with kinetic energies many times greater than $240 \mathrm{MeV}$ Jokipii and Kota (1995) have also recently suggested that increased latitudinal diffusion can help explain how ACRs get accelerated to $>1 \mathrm{GeV}$.

Both SAMPEX (Mewaldt et al. 1993) and Geotail (Hasebc st al., 1994) investigators have reported a "bump" in the 1992.1993 carbon spectrum at $\sim 10$ to $15 \mathrm{MeV} /$ nuc that was $\sim 5$ times more intense than expected for ACR carbon, giving a $\mathrm{C} / \mathrm{O}$ ratio of $\sim 0.1$. Although the MAST data are limited Io carbon with $>14 \mathrm{MeV} /$ nuc, Figures 2 and 3 (and Table 1) indicate a $\mathrm{C} / \mathrm{O}$ ratio at mid-latitudes similar to that observed at Voyager, indicating that the 1992-1993 feature in the carbon spectrum was apparently not due to singly-ionized carbon.

Biswas et al. (1990; see also Dutta et al. 1993) have reported Spacelab-3 observations of heavy nuclei with $21 \leq \mathrm{Z} \leq 28$ that were apparently partially stripped, since they were observed at latitudes inaccessible to fully stripped cosmic rays. They concluded that $\sim 20 \%$ of heavy GCRs with $<100 \mathrm{MeV} / \mathrm{nuc}$ observed during their 1985 flight were partially ionized (Dutta et al. 1993). We see no stiong evidence for the presence of these particles in 1992-1994, when the vast majority of cosmic ray nuclei with $Z \geq 12$ appear to be fully stripped (see also Tylka et al. 1995). If we assume, for example, that $20 \%$ of the GCRs in the SAMPEX energy 
range are partially stripped nuclei with $Q / Z<1 / 4$, we would expect to have observed $>100$ mid-latitude events with $Z \geq 12$, compared to the three candidates in Figure 2. Mewaldt (1995) has suggested that the apparent "partially stripped GCRs" might be solar particles re-accelerated at the termination shock, which should be more abundant during the Spacelab-3 period than in 1992-1994 (although this explanation would not explain the apparently large fraction of nuclei with $22 \leq \mathrm{Z} \leq 24$ reported by Dutta et al.). In addition, Tylka et al. (1995) have suggested a cosmic ray albedo origin.

The observations presented here demonstrate that the geomagnetic filter approach can successfully obtain a pure sample of ACR ions. This approach will be particularly useful for measuring the isotopic composition of ACR nuclei as a means of studying the isotopic composition of the nearly interstellar medium (for preliminary results see Leske ct al. 1995b). In addition, the fact that the ACR cnergy spectrum extends to $\sim 1.6 \mathrm{GeV}$, when combined with thcuretical considerations, suggests that the highest energy ACRs may not be singly-charged. A possible test of this hypothesis using SAMPEX data is now in progress.

Acknowledgments: This work was supported by NASA uncler NAS5-30704 \& NAGIV-1919. We appıeciate discussions with A. C. Cummings.

\section{References}

Adams, J. H. Jr., \& M. D. Leising, Maximum distance to the acceleration site of the anomalous component of cosmic rays, Proc. 22nd Internat. Cosmic Ray Conf. (Dublin) 3, 304, 1991.

Adams, J. H. Jr. et al., The charge state of the anomalous component of cosmic rays, Astrophys. J. 375, L45, 1991

Baker, D. N., et al., An overview of the Solar, Anomalous, and Magnetospheric Particle Explorer (SAMPEX) mission, IEEE Trams. Geosci. Remote Sensing 31, 531, 1993.

Blake, J. B., and L. M. Friesen, A technique to determine the charge state of the anomalous low-energy cosmic rays, Proc. J5th Int'tnat. Cosmic Ray Conf. (Plovdiv) 2, 341, 1977.

Biswas, S., et al., Observation of low-energy (30-100 MeV nucleon ${ }^{-1}$ ) partially ionized heavy ions in galactic cosmic rays, Astrophys. I. 359, L5, 1990.

Cook, W. R., et al., MAST: A Mass Spectrometer Telesiopu for studi es of the isotopic composition of solar, anomalous, and galactic cosnse ray nuclei, IEEE Trans. Geosci. Remote Sensing 31, 557, 1993.

Cummings, A. C., and E. C. Stone, Composition, gradients, and temporai variations of the anomalous cosmic ray component, Pruc. Sixth Internat. Solar Wind Conf. V J. Pizzo, T. E. Holzer, and D. G, Sime, eds., NCAR Technical Note TN-306 2, 599, 1988.

Cummings, J. R., ct al., New evidence for geomagnetically trapped anomalous cosmic rays, Geophys. Res. Lett. 20, 2003, 1993.
Dutta, A., et al.. Ionization states of low-energy cosmic rays: results from Spacelab 3 cosmic ray experiment, Astroplys J. 4lI, 418, 1993.

Fisk, L. A., B. Kozlovsky, and R. Ramaty, An interpretation of the observed oxygen and nitrogen enhancements in low-energy cosmuc rays, Astrophys J. /90, L35, 1974.

Grigorov, N., et al., Evidence for anomalous cosmic ray oxygen ions in the inner magnetosphere, Geophys. Res. Lett 18, 1959, 1991.

Hasebe, N., et al., Rapid recovery of anomalous cosmic ray flux at 1 AU in solar cycle 22, Geophys Res. Lett 21, 3027, 1994.

Jokipii, J. R., The anomalous component of cosnic rays, in P/hysis s of the Outer Heliosphere, S. Grzedzielskı and D. E. Page, Pergaumon Press 1990.

Jokipii, J. R., Constraints on the acceleration of anomalous cosmıc ıays, Astrophys J. 393, L41, 1992.

Jokipii, J. R., and J. Kota, The maximum energy of anomalous cosnic rays, Proc. 24th Internat. Cosmic Ray Conf. (Rome) 4, 718, 1995

Klecker, B., The anomalous component of cosmic rays in the 3-D heliosphere, Space Science Reviews 72, 419, 1995.

Klecker, B., et al., Charge state of anomalous cosinic ray nitrogen, oxygen, and neon: SAMPEX observations, Astrophys J. 442, L69, 1995.

Leske, R. A., et al., Measurements of the ionic charge states of solar energetic particles at $15-70 \mathrm{MeV} /$ nucleon, Proc. 24th Internat. Cosmic Ray Conf. (Rome) 4, 461, 1995a.

Leske, R. A., et al., The isotopıc composition of anomalous and galactic cosmic rays from SAMPEX, Proc. 24th Internat. Cosmic Ray Cont. (Rome) 2, 606, $1995 \mathrm{~b}$.

Luhn, A., et al., Ionic charge states of $\mathrm{N}, \mathrm{Ne}, \mathrm{Mg}, \mathrm{Si}$, and $\mathrm{S}$ in solar energetic particle events, Adv. Space Res. 4, No. 2-3, 161, 1984.

Mewaldt, R. A., et al., The return of the anomalous cosinic rays to I AU in 1992, Geophys. Res. Lett. 20, 2263,1993.

Mewaldt, R. A., Solar and interplanetary particles re-accelerated at the solar wind termination shock, Proc. 24th Internat. Cosmic Ray Conf (Rome) 4, 804, 1995.

Mewaldt, R. A., et al., Studies of anomalous cosmic rays using the geomagnetic field, Proc. 24th Internat. Cosmic Ray Conf. (Rome) 4, $477,1995$.

Pesses, M. E., J. R. Jokipii, and D. Eichler, Cosmic ray drift, shock wave acceleration, and the anomalous component of cosmic rays, Astrophys. J. 246, L85, 1981.

Selesnick, R. S., et al., Geomagnetically trapped anomalous cosmic rays, J. Geophys. Res. 100, 9503, 1995.

Simpson, J. A., The anomalous nuclear component in the threedimensional heliosphere, to be published in Advances in Space Research 1995.

Tylka, A. J., P. R. Boberg, J. H. Adams, Jr., T. Kleis, and R. Beaujean, On purported observations of partially ionized galactic cosmic rays, Astrophys. J. 438, L83, 1995.

J. R. Cummings, R. A. Leske, R. A. Mewaldt, R. S. Selesnick, and E. C. Stone, 220-47 Caltech, Pasadena, CA 91125. dick@ srl.caltech.edu T. T. von Rosenvinge, GSFC Code 661, Greenbelt, MD 20771.

(Received: August 28, 1995; revised: October 17, 1995; accepted October 23, 1995) 